\title{
Effects of tracheotomy on respiratory mechanics in spontaneously breathing patients
}

\author{
V. Moscovici da Cruz*, S.E. Demarzo*, J.B.B. Sobrinho", M.B.P. Amato\#, L.P. Kowalski*, \\ D. Deheinzelin*
}

Effects of tracheotomy on respiratory mechanics in spontaneously breathing patients. V. Moscovici da Cruz, S.E. Demarzo, J.B.B. Sobrinho, M.B.P. Amato, L.P. Kowalski, D. Deheinzelin. C ERS Journals Ltd 2002.

ABSTRACT: Tracheotomy is a method of intubating the trachea, which is employed in several clinical settings, including the treatment of head and neck neoplasms. Tracheotomy is believed to facilitate weaning through changes in respiratory mechanics. Existing information concerning functional changes associated with tracheotomy are limited to comparisons with orotracheal intubation.

In this study, respiratory mechanics were monitored in seven spontaneously breathing patients, before and after an elective tracheotomy was performed for surgical treatment of cancer. Campbell diagrams were constructed by plotting pressure, obtained with an oesophageal balloon catheter, against volume, obtained from a pneumotachograph placed at the airway opening. Work of breathing was calculated as the internal area of the Campbell diagram and was partitioned into its elastic and inspiratory and expiratory resistive components. Oesophageal pressure was also used to quantify intrinsic positive end-expiratory pressure (PEEPi) and the pressure-time product (PTP), which is considered to be proportional to the oxygen cost of breathing. PTP was divided into its resistive and elastic components.

Inspiratory resistive work, PEEPi, inspiratory PTP, as well as its resistive and elastic components were significantly reduced by tracheotomy.

Tracheotomy significantly reduces work of breathing and pressure-time product in spontaneously breathing patients.

Eur Respir J 2002; 20: 112-117.

\begin{abstract}
*Centre for Treatment and Research, Cancer Hospital, Antonio Prudente Foundation and ${ }^{\sharp}$ Faculty of Medicine, University of São Paulo, São Paulo, Brazil.
\end{abstract}

Correspondence: V. Moscovici da Cruz, Unidade de Terapia Intensiva, Hospital do Câncer, Rua Prof. Antonio Prudente, $2116^{\circ}$ andar, São Paulo SP, Brasil, CEP 01509-010.

Fax: 551130219787

E-mail: vasco77@hotmail.com

Keywords: Intrinsic positive-pressure respiration, physiopathology, respiratory mechanics, tracheotomy, work of breathing

Received: August 162001

Accepted after revision: January 142002

This study was supported by grants from the Fundação de Amparo à Pesquisa do Estado de São Paulo (FAPESP).
Tracheotomy is a standardised and widespread procedure [1], however, its functional implications have been not been exhaustively studied. Tracheotomy is indicated in several clinical conditions, including relief of upper airway obstruction by neoplasms, inflammation or trauma, and is also performed on patients under prolonged mechanical ventilation, where it is believed to facilitate weaning [2].

Viewed from a respiratory standpoint, the physiological implications of tracheotomy are related to loss of upper airway and laryngeal function and resistance of the artificial airway. In patients with pulmonary disease these implications may be more important.

In a recent study, DieHl et al. [3] studied mechanically ventilated patients submitted to tracheotomy because of prolonged intubation, and observed a reduction in the mechanical work of breathing (WOB) and intrinsic positive end-expiratory pressure (PEEPi) after the procedure, when compared to the condition before with an orotracheal tube. Under normal circumstances, the larynx represents a small fraction of total airflow resistance $[4,5]$. However, the transition from orotracheal tube to tracheotomy cannula has a significant impact on PEEPi and mechanical WOB.
These observations suggest that it is the reduction in airway length that explains the observed variations.

The study by DiEHL et al. [3] did not evaluate spontaneously breathing nonintubated patients, as is the case in surgically treated head and neck cancer patients and individuals who have been mechanically ventilated and have been successfully weaned. Previous studies [6, 7] have reported the presence of PEEPi in stable, nonintubated chronic obstructive pulmonary disease patients. Loss of laryngeal function has not been addressed previously.

The Head and Neck Surgery Dept of the São Paulo Cancer Hospital, where this study was conducted, performs around 200 annual elective procedures that include tracheotomy. The aim of the present study was to functionally assess the mechanics of breathing of stable patients submitted to elective tracheotomy.

\section{Methods}

The study was approved by the local ethics committee, and all patients involved gave informed consent. Analysis of pressure/volume relationships was 
used to assess changes in respiratory mechanics imposed by tracheotomy.

Variations in oesophageal pressure $\left(P_{\mathrm{oes}}\right)$ variation correlate well with variations in pleural pressure [8]. Therefore, Poes was measured using an oesophageal balloon catheter (SmartCath; Allied Healthcare, Irvine, CA, USA) with a $0.8 \mathrm{~mL}$ inflation volume automatically delivered by a Bicore CP-100 (Allied Healthcare) pulmonary monitor. Measurements were validated before and after surgery using the technique described by BAYDUR et al. [9].

Flow values were obtained using a variable orifice pneumotachograph (VarFlex Flow Transducer; Allied Healthcare) held between the patient's lips. The oesophageal catheter and the pneumotachograph were connected to a Bicore CP-100 pulmonary monitor. Nostrils were occluded by a noseclip. Measurements were performed with patients in the sitting position before and after surgery.

The oesophageal catheter was kept in position during the surgical procedure. A new measurement was taken on the first day after surgery, when the patient was awake and had not required ventilatory support for at least $12 \mathrm{~h}$. For this measurement the pneumotachograph was connected to a cuffed tracheotomy tube (Portex tubes with 7 or $8 \mathrm{~mm}$ internal diameter).

Variables were recorded in a notebook computer, using the Lab View 5.0 (National Instruments, Austin, TX, USA) program. The program generated text files relative to time from tracheal pressure, oesophageal pressure and flow. Acquisition frequency was $100 \mathrm{~Hz}$. Pre- and postoperative measurements lasted $5 \mathrm{~min}$. Using files developed at the Discipline of Pulmonology of the Faculty of Medicine of the University of São Paulo, the onset of each respiratory cycle was determined and on-screen visualisation allowed exclusion of spurious cycles (that could correspond to swallowing or cough) which differed from the predominant pattern. A mean cycle was then calculated to minimise artefacts.

Mean cycles were exported to Quatro Pro 7 (Corel Word Perfect Suite; Corel Corporation, Ottawa, Ontario, Canada) where the WOB and pressure-time product (PTP) were estimated from the area under the pressure/volume (Campbell diagram [10]) and pressure/times curves [11], respectively. The lung compliance line $(C \mathrm{~L}$,dyn) was plotted on the Campbell diagram by linking end-inspiratory and end-expiratory points of zero flow. Chest-wall compliance $(C \mathrm{w})$ was not measured, since not all patients were routinely paralysed, but was estimated to be equal to $4 \%$ of the predicted forced vital capacity (FVC). Therefore, to avoid possible chest-wall compliance alterations induced by anaesthesia and surgical procedure, measurements were obtained within intervals $>12 \mathrm{~h}$ before or after the operations.

PEEPi was measured as the maximal negative deflection of $P_{\text {oes }}$ at the onset of inspiration, in which no volume increment was observed. This approach assumed no expiratory muscle activity. However, this deflection could be interpreted either as the effort necessary to overcome PEEPi or, assuming absence of $\mathrm{PEEPi}$, the relaxation of expiratory muscles activated during expiration. To avoid this problem, the $C_{\mathrm{w}}$ line was positioned on the Campbell diagram according to both situations, as described previously as upper bound and lower bound, respectively [12].

WOB was calculated separately in its elastic (WOBel) and inspiratory-resistive (WOBri) components. WOBel was defined by the area between the two compliance lines $(C \mathrm{~L}$, dyn and $C w)$ and limited by the maximum volume achieved. WOBel was analysed considering upper and lower bound situations separately, as described above. WOBri was defined by the area inside the pressure/volume loop to the left of $C \mathrm{~L}$,dyn. Therefore, WOBri corresponds to the inspiratory phase of the respiratory cycle. WOB values were expressed as joules $(\mathbf{J}) \cdot \mathrm{L}^{-1}$.

PTP was calculated similarly considering upper and lower bound situations and by plotting a theoretical chest-wall elastic recoil pressure $\left(P_{\text {oes, }}, \mathrm{CW}\right)$ curve according to the following equation:

$$
P_{\text {oes }, \mathrm{CW}}=\left(E_{\text {dyn }, \mathrm{CW}} \times V_{\mathrm{L}}\right)+P_{\text {oes, }, \mathrm{CW}, \text { eep }}
$$

where $E$ dyn, $C W$ is the chest-wall dynamic elastance (the reciprocal of $C w$, estimated as $4 \%$ of predicted FVC), $V \mathrm{~L}$ is the lung volume above the end-expiratory volume and $P_{\text {oes, }} \mathrm{CW}$,eep is the $P_{\text {oes }}$ at the point of transition between the end of expiration and the beginning of inspiration. Upper bound PTP assumes that $P_{\text {oes }}, \mathrm{CW}$,eep equals $P_{\text {oes }}$ when it undergoes a sharp negative deflection before the beginning of inspiratory flow, while lower bound PTP assumes $P$ oes, CW,eep as equal to $P$ oes at the onset of inspiratory flow. PTP is expressed in $\mathrm{cmH}_{2} \mathrm{O} \cdot \mathrm{s}^{-1}$.

The resistive component of PTP (PTPr) was calculated as the area under the line of lung recoil pressure $(P$ oes,L) in the pressure/time curve, the curve of which was plotted according to the following formula:

$$
P_{\text {oes }, \mathrm{L}}=\left(E_{\text {dyn }, \mathrm{L}} \times V_{\mathrm{L}}\right)+P_{\text {oes, } \mathrm{L}, \text { eep }}
$$

where $E$ dyn,L is the calculated lung elastance (the reciprocal of $C$ dyn, I as obtained from the Campbell diagram), $V \mathrm{~L}$ is the lung volume above the endexpiratory volume and $P$ oes,L,eep is the oesophageal pressure at the onset of inspiratory flow.

The elastic component (PTPel) was calculated as the integral of the area of the pressure/time curve limited by the lung elastic-recoil curve and the chest-wall recoil pressure line upper bound. The elastic component, due to the presence of PEEPi, was calculated as the integral of the area limited by lower- and upperbound derived chest-wall recoil pressure lines (fig. 1).

Variables were found to have a nonparametric distribution, and were compared using the Wilcoxon test.

\section{Results}

Twenty-three patients were eligible for the study. Eight patients refused to participate, in four patients progression of the oesophageal-balloon catheter to the necessary position for oesophageal-pressure monitoring was impossible, one patient lost the catheter 


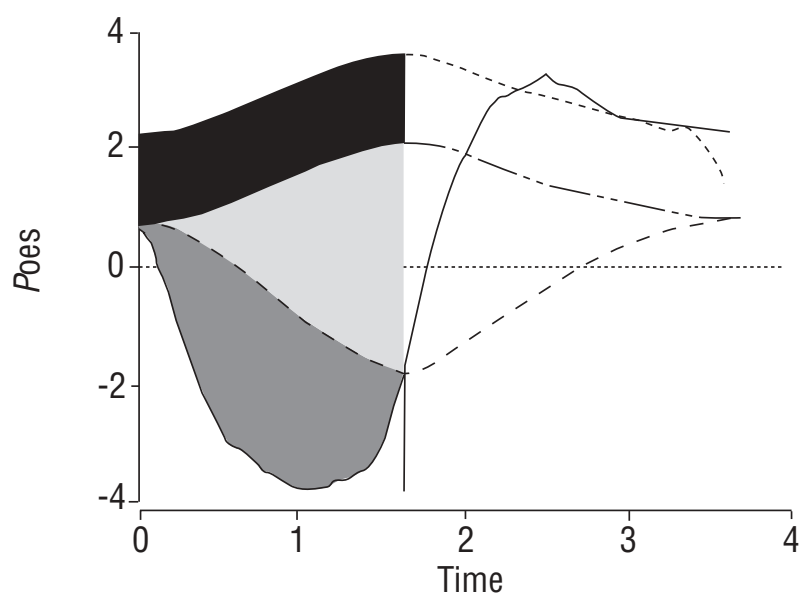

Fig. 1.- Pressure/time curve showing upper bound (---) and lower bound (-.-) chest wall recoil pressure, and lung recoil pressure $\left(-{ }_{-}\right)$. Poes: oesophageal pressure; t: time; $\mathbf{\square}$ : elastic pressure-time product (PTP) relative to instrinsic positive endexpiratory pressure $\left(\mathrm{PEEP}_{\mathrm{i}}\right)$; : elastic PTP unrelated to PEEPi; : resistive PTP.

during surgery and therefore postoperative values could not be obtained, one patient had surgery cancelled and two patients had clinical syndromes at hospital admission (psychotic symptoms and alcoholic intoxication) that rendered the procedures necessary for the study impossible. Therefore, data was obtained pre- and postoperatively for seven patients.

Patient demographics are shown in table 1. Figure 2 shows the Campbell diagrams obtained from $P_{\text {oes }}$ and volume measurements of the mean cycles pre- and postoperatively for the seven patients.

Postoperative Campbell diagrams obtained from patients 1, 2, 4, 5, 6 and 7 showed loss of the configuration indicative of the presence of PEEPi.

Results of inspiratory resistive work, elastic work and PEEPi from all patients before and after surgery are shown in tables 2 and 3. Tables 4 and 5 show results PTP and its components before and after surgery. The main results were a significant reduction of PEEPi as well as a reduction of all components of PTP, except for PTP lower bound, and a significant reduction in WOBel. $V \mathrm{~T}$ did not change significantly pre- and postoperatively $(0.38 \pm 0.17$ and $0.40 \pm 0.11$, $\mathrm{p}=0.610)$ while respiratory rate increased significantly $(15 \pm 3$ and $20 \pm 6, \mathrm{p}=0.04)$.

\section{Table 1. - Patient demographics}

\begin{tabular}{lccc}
\hline Patient no. & Sex & Age yrs & Tumour location $^{\#}$ \\
\hline 1 & M & 61 & Tonsil \\
2 & M & 45 & Palate \\
3 & M & 57 & Base of tongue \\
4 & F & 45 & Base of tongue \\
5 & M & 64 & Larynx \\
6 & M & 86 & Tongue \\
7 & M & 57 & Larynx \\
\hline
\end{tabular}

M: male; F: female; \#: All patients had squamous-cell carcinoma.
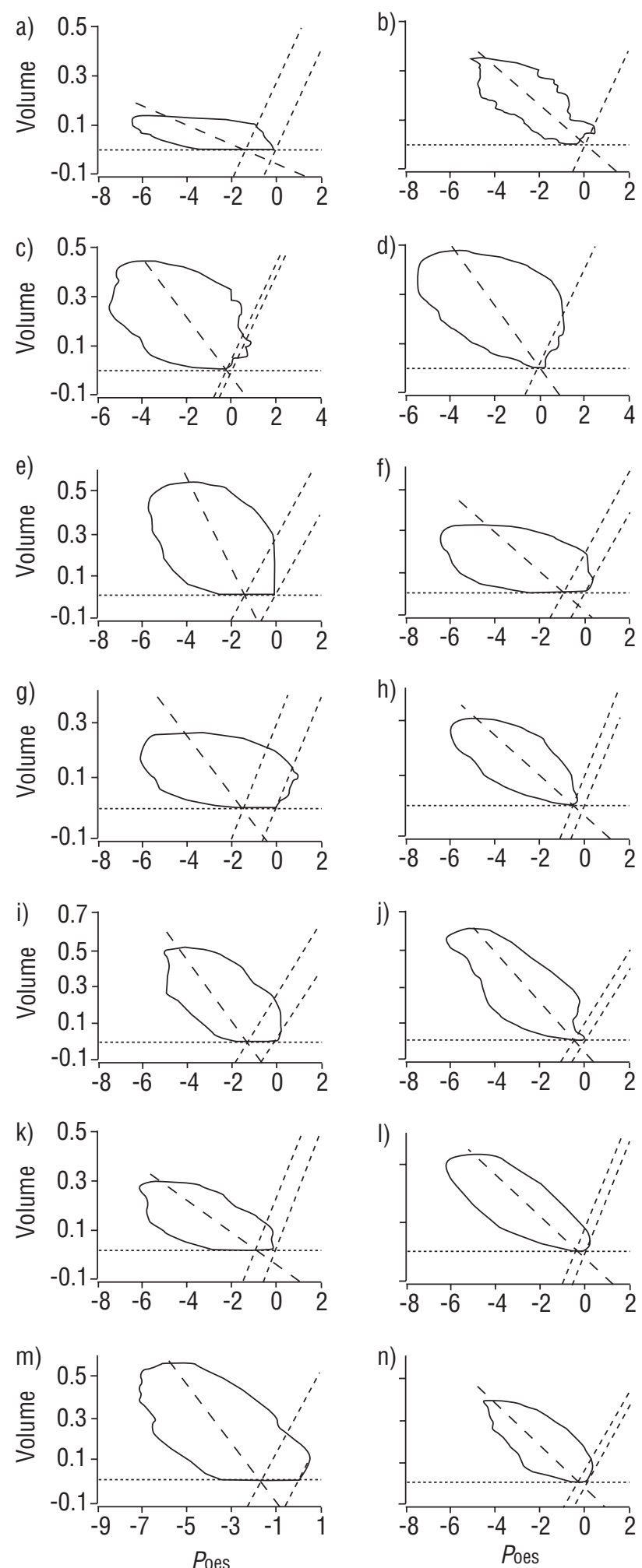

Fig. 2.-Campbell diagrams for patients 1 (a and b), 2 (c and d), 3 (e and $\mathrm{f}), 4$ (g and $\mathrm{h}$ ), 5 ( $\mathrm{i}$ and $\mathrm{j}$ ), 6 (k and $\mathrm{l}$ ) and $7(\mathrm{~m}$ and $\mathrm{n})$. The left column shows the pre-operative diagrams and the right column shows the postoperative diagrams. Chest wall compliance lines (----) are shown for both situations: upper bound on the right and lower bound on the left. Dynamic lung compliance lines $(---)$ are also shown. Postoperative diagrams from patients 1 and 2 show near superposition of the two lines. 
Table 2. - The resistive and elastic components of work of breathing (WOB) obtained before and after tracheotomy

\begin{tabular}{|c|c|c|c|c|c|c|}
\hline \multirow[b]{2}{*}{ Patient no. } & \multicolumn{2}{|c|}{ WOBri $\mathbf{J} \cdot \mathrm{L}^{-1}$} & \multicolumn{2}{|c|}{ WOBel $\mathbf{J} \cdot \mathrm{L}^{-1}$} & \multicolumn{2}{|c|}{$\mathrm{WOBel}^{\prime} \mathrm{J} \cdot \mathrm{L}^{-1}$} \\
\hline & Pre & Post & Pre & Post & Pre & Post \\
\hline 1 & 0.84 & 0.11 & 0.21 & 0.32 & 0.35 & 0.32 \\
\hline 2 & 0.37 & 0.30 & 0.30 & 0.32 & 0.33 & 0.32 \\
\hline 3 & 0.34 & 0.26 & 0.27 & 0.26 & 0.48 & 0.39 \\
\hline 4 & 0.34 & 0.64 & 0.22 & 0.33 & 0.42 & 0.39 \\
\hline 5 & 0.49 & 0.24 & 0.31 & 0.40 & 0.49 & 0.48 \\
\hline 6 & 0.64 & 0.21 & 0.30 & 0.37 & 0.41 & 0.41 \\
\hline 7 & 0.37 & 0.14 & 0.37 & 0.31 & 0.61 & 0.34 \\
\hline Mean \pm SD & $0.48 \pm 0.19$ & $0.27 \pm 0.18$ & $0.28 \pm 0.06$ & $0.33 \pm 0.05$ & $0.44 \pm 0.10$ & $0.38 \pm 0.06$ \\
\hline $\mathrm{p}$-value & & 0.13 & & 0.11 & & 0.03 \\
\hline
\end{tabular}

WOBri: resistive work of breathing; WOBel: elastic work of breathing considering the lower bound situation; WOel': elastic work of breathing considering the upper bound situation.

\section{Discussion}

The results of this study showed a significant reduction in mechanical-work parameters after tracheotomy in spontaneously breathing nonintubated patients. This trend is demonstrated best in data obtained from the PTP measurements. Oxygen consumption has been demonstrated to correlate with PTP better than with mechanical-work measurements [13] as time, a determinant of oxygen consumption by muscle, is not taken into account in WOB calculations, as it is in PTP.

Previous work $[14,15]$, using Bicore crude values to

Table 3. - Intrinsic positive end-expiratory pressure (PEEPi) obtained before and after tracheotomy for each patient

\begin{tabular}{ccc}
\hline & \multicolumn{2}{c}{ PEEPi $\mathrm{cmH}_{2} \mathrm{O}$} \\
\cline { 2 - 3 } Patient no. & Pre & Post \\
\hline & & \\
1 & 1.34 & 0.00 \\
2 & 0.18 & 0.01 \\
3 & 1.28 & 1.00 \\
4 & 1.44 & 0.50 \\
5 & 1.26 & 0.51 \\
6 & 0.85 & 0.35 \\
7 & 1.72 & 0.28 \\
Mean \pm SD & $1.15 \pm 0.50$ & $0.38 \pm 0.34$ \\
p-value & & 0.02 \\
\hline
\end{tabular}

compare ventilation through an orotracheal tube and through tracheotomy, failed to demonstrate any reduction in WOB parameters. In the current authors view this was mainly due to the methods employed.

The use of the mean cycle as the source for calculating work values minimised artefacts and allowed the visualisation of a Campbell diagram representative of a long period of observation time. Moreover, it allowed the exclusion of spurious cycles caused by swallowing or coughing, and the treatment of the signals obtained allowed a more accurate scrutiny of the different components of WOB and PTP. SPAHIJA et al. [16] used mean cycles to deal with the mechanics of breathing experimentally and suggested that this procedure should be applied to clinical practice.

The current authors assumed that the same theoretical value of $C_{\mathrm{w}}$ could be used to compare the same patient before and after tracheotomy. Direct measurement of $C_{\mathrm{w}}$ would certainly yield different numerical values, but as this study compared the same patient at two different times, the difference would remain.

Using an similar approach DiEHL et al. [3] demonstrated a reduction in work parameters after tracheotomy in mechanically ventilated patients, in relation to the endotracheal tube. To the best of the authors' knowledge the present report is the first to demonstrate benefit in nonintubated patients.

Results have been expressed considering two extreme situations, upper bound and lower bound. These

Table 4. - Pressure-time product (PTP) upper bound, PTP lower bound, and PTP due to intrinsic positive end-expiratory pressure (PTPI)

\begin{tabular}{|c|c|c|c|c|c|c|}
\hline \multirow[b]{2}{*}{ Patient no. } & \multicolumn{2}{|c|}{ PTP upper bound } & \multicolumn{2}{|c|}{ PTP lower bound } & \multicolumn{2}{|c|}{ PTPi } \\
\hline & Pre & Post & Pre & Post & Pre & Post \\
\hline 1 & 8.19 & 7.09 & 6.21 & 7.08 & 1.98 & 0.00 \\
\hline 2 & 10.40 & 6.49 & 10.06 & 6.47 & 0.34 & 0.02 \\
\hline 3 & 12.69 & 6.26 & 10.02 & 5.27 & 2.67 & 0.98 \\
\hline 4 & 9.30 & 4.66 & 6.96 & 4.22 & 2.35 & 0.44 \\
\hline 5 & 10.13 & 9.75 & 7.88 & 8.96 & 2.25 & 0.78 \\
\hline 6 & 7.18 & 6.08 & 6.17 & 5.69 & 1.01 & 0.39 \\
\hline 7 & 19.35 & 8.51 & 15.13 & 8.01 & 4.23 & 0.49 \\
\hline Mean \pm SD & $11.04 \pm 4.06$ & $6.97 \pm 1.68$ & $8.92 \pm 3.19$ & $6.53 \pm 1.64$ & $2.12 \pm 1.24$ & $0.44 \pm 0.36$ \\
\hline p-value & & 0.02 & & 0.13 & & 0.02 \\
\hline
\end{tabular}


Table 5. - Resistive pressure-time product (PTPr) and elastic PTP (PTPel)

\begin{tabular}{ccccc}
\hline & \multicolumn{2}{c}{ PTPr } & \multicolumn{2}{c}{ PTPel } \\
\cline { 2 - 3 } Patient & Pre & Post & Pre & Post \\
\cline { 2 - 4 } 1 & 2.92 & 2.07 & 5.27 & 5.01 \\
2 & 4.15 & 2.50 & 6.26 & 3.99 \\
3 & 4.21 & 2.59 & 8.48 & 3.66 \\
4 & 3.47 & 1.31 & 5.84 & 3.35 \\
5 & 2.25 & 2.42 & 7.88 & 7.32 \\
6 & 2.25 & 1.83 & 14.45 & 4.24 \\
7 & 4.90 & 1.67 & $7.59 \pm 3.30$ & 6.84 \\
Mean \pm SD & $3.45 \pm 1.03$ & $2.06 \pm 0.48$ & & $4.92 \pm 1.57$ \\
p-value & & 0.03 & & 0.02 \\
\hline
\end{tabular}

situations occur considering that either the negative deflection of the pressure curve as due only to PEEPi (upper bound) or in the complete absence of PEEPi, considering the negative deflection observed in the pressure curve as due solely to expiratory muscle activity (lower bound). In fact it is theoretically possible that during any respiratory cycle a patient is in an intermediate situation between these extremes. The observations by CAMPBELL and FRIENDS [17] showed that under expiratory muscle activity, $P_{\text {oes }}$ increases during expiration. Such a pattern could be seen in only one of the patients in this study (no. 4). Thus, the current authors believe that the upper bound values correctly describe the mechanical alterations observed in the patients studied. Whatever the option, a graphical change in the Campbell diagram was observed in all patients.

Expiratory muscle activity can be alternatively observed through the measurement of gastric pressure. Recent work has shown that a large part of the pressure change measured as PEEPi can be attributed to expiratory muscle activity [18]. Unfortunately, gastric pressure was not measured here and therefore the real cause of the changes observed cannot be determined. Nonetheless, a parameter independent of whether expiratory muscle activity was considered or not, i.e. upper or lower bound, namely the resistive component of PTP, was shown to decrease significantly.

Could the functional benefit observed be due to the relief of tumour-induced upper airway obstruction in this population? Only three of seven patients had tumours in structures that could be implicated in airway obstruction (larynx and tonsil), and all others exhibited numerical and graphic changes, thus the authors believe that these findings are consistent with benefit irrespective of upper airway obstruction. Similarly, observation of preoperative flow/volume curves obtained from the mean cycles (data not shown) did not disclose the usual pattern associated with variable extrathoracic obstruction. In spite of a relatively small number of patients who accepted the insertion of a naso-oesophageal catheter on the evening before surgery, consistent evidence was obtained supporting the hypothesis that tracheotomy reduces the work of breathing, probably by decreasing resistance and intrinsic positive end-expiratory pressure.

\section{References}

1. Wenig BL, Applebaum EL. Indications for and techniques of tracheotomy. Clin Chest Med 1991; 12: $545-553$.

2. Heffner JE, Miller KS, Sahn SA. Tracheostomy in the intensive care unit: Part 1. Indications, technique, management. Chest 1986; 90: 269-274.

3. Diehl JL, Atrous SE, Touchard D, Lemaire F, Brochard L. Changes in the work of breathing induced by tracheotomy in ventilator-dependent patients. $\mathrm{Am}$ J Respir Crit Care Med 1999; 159: 383-388.

4. Schiratzki H. The oral and laryngeal components of the upper airway resistance during mouth breathing. Acta Oto-Laryngol 1965; 60: 71-82.

5. Spann RW, Hyatt RE. Factors affecting upper airway resistance in conscious man. J Appl Physiol 1971; 31: 708-712.

6. Dal Vecchio L, Polese G, Poggi R, Rossi A. "Intrinsic" positive en-expiratory pressure in stable patients with chronic obstructive pulmonary disease. Eur Respir $J$ 1990; 3: 74-80.

7. Haluszka J, Chartrand DA, Grassino AE, Milic-Emili $\mathrm{J}$. Intrinsic PEEP and arterial $\mathrm{pCO} 2$ in stable patients with chronic obstructive pulmonary disease. Am Rev Respir Dis 1990; 141: 1194-1197.

8. Milic-Emili J, Mead J, Turner JM, Glauser EM. Improved technique for estimating pleural pressure from esophageal balloons. J Appl Physiol 1964; 19: 207-211.

9. Baydur A, Behrakis PK, Zin WA, Jaeger MJ, MilicEmili J. A simple method for assessing the validity of the esophageal balloon technique. Am Rev Respir Dis 1982; 126: 788-791.

10. Campbell EJM. The respiratory muscles and the mechanics of breathing. Chicago, IL, Year Book Medical, 1958.

11. Tobin MJ. Monitoring respiratory mechanics in spontaneously breathing patients. In: Tobin MJ, ed. Principles and Practice of ICU Monitoring. McGrawHill, New York, 1998; pp. 617-654.

12. Jubran A, Tobin MJ. Pathophysiological basis of acute respiratory distress in patients who fail a trial of weaning from mechanical ventilation. Am J Respir Crit Care Med 1997; 155: 906-915.

13. Field S, Sanci S, Grassino A. Respiratory muscle oxygen consumption estimated by the diaphragm pressure-time index. J Appl Physiol 1984; 57: 44-51.

14. Lin MC, Huang CC, Yang CT, Tsai YH, Tsao TCY. Pulmonary mechanics in patients with prolonged 
mechanical ventilation requiring tracheotomy. Anaesth Intensive Care: 1999: 581-585.

15. Davis KJ, Campbell RS, Johannigman JA, Valente $\mathrm{JF}$, Branson RD. Changes in respiratory mechanics after tracheotomy. Arch Surg 1999; 134: 59-62.

16. Spahija JA, Grassino A. Effects of pursed-lips breathing and expiratory resistive loading in healthy subjects. J Appl Physiol 1996; 80: 1772-1784.
17. Campbell E, Friend J. Action of breathing exercises in pulmonary emphysema. Lancet 1955; 1: 325329.

18. Lessard MR, Lofaso F, Brochard L. Expiratory muscle activity increases intrinsic positive endexpiratory pressure independently of dynamic hyperinflation in mechanically ventilated patients. $\mathrm{Am}$ J Respir Crit Care Med 1995; 151: 562-569. 